\title{
Using the Albumin-Bilirubin (ALBI) grade as a prognostic marker for radioembolization of hepatocellular carcinoma
}

\author{
Homan Mohammadi ${ }^{1}$, Yazan Abuodeh ${ }^{1}$, William Jin ${ }^{2}$, Jessica Frakes ${ }^{1}$, Mark Friedman ${ }^{3}$, Benjamin Biebel ${ }^{3}$, \\ Junsung Choi ${ }^{3}$, Ghassan El-Haddad ${ }^{3}$, Bela Kis ${ }^{3}$, Jennifer Sweeney ${ }^{3}$, Sarah Hoffe ${ }^{1}$ \\ ${ }^{1}$ Department of Radiation Oncology, Moffitt Cancer Center, Tampa, FL, USA; ${ }^{2}$ Morsani College of Medicine, University of South Florida, Tampa, \\ FL, USA; ${ }^{3}$ Department of Gastroenterology, Moffitt Cancer Center, Tampa, FL, USA \\ Contributions: (I) Conception and design: S Hoffe, J Frakes, W Jin, H Mohammadi; (II) Administrative support: S Hoffe, J Frakes; (III) Provision of \\ study material or patients: M Friedman, B Biebel, J Choi, G El-Haddad, B Kis, J Sweeney, S Hoffe, J Frakes; (IV) Collection and assembly of data: H \\ Mohammadi, Y Abuodeh, J Frakes; (V) Data analysis and interpretation: H Mohammadi, W Jin, Y Abuodeh, S Hoffe; (VI) Manuscript writing: All \\ authors; (VII) Final approval of manuscript: All authors. \\ Correspondence to: Homan Mohammadi, MD. 12902 Magnolia Drive, Tampa, FL 33612, USA. Email: Homan.Mohammadi@Moffitt.org.
}

\begin{abstract}
Background: The Child-Pugh (CP) class is a commonly used scoring system to measure liver function in patients with hepatocellular carcinoma (HCC). We correlate the Albumin-Bilirubin (ALBI) grading system and CP to overall survival in our HCC patients receiving radioembolization.

Methods: We retrospectively evaluated patients who received radioembolization for HCC between the years 2009-2014. We evaluated the albumin and bilirubin levels in our patients prior to receiving their first $(n=124)$ radioembolization. The ALBI grades were calculated from these data with the formula $\left(\log _{10}\right.$ bilirubin $\times 0.66)+($ albumin $\times-0.085)$ and correlated to outcomes using Mantel-Cox Log analysis. These statistical comparisons were duplicated with CP classes.

Results: Median survival differences between CP class A and B and between ALBI grade 1 and 2 were 4.7 and 9.9 months, respectively. A subset of ALBI grades 1 and 2 were identified within our CP class A patients with a median survival difference of 9.9 months.

Conclusions: ALBI is a more sensitive marker of liver function than CP in the setting of mild dysfunction. Using ALBI, we identified a subset of patients that have significantly better outcomes from Y-90 radioembolization than previously identified with $\mathrm{CP}$.
\end{abstract}

Keywords: Hepatocellular carcinoma (HCC); radioembolization; Albumin-bilirubin (ALBI); Child-Pugh (CP)

Submitted Apr 05, 2018. Accepted for publication May 13, 2018.

doi: 10.21037/jgo.2018.05.14

View this article at: http://dx.doi.org/10.21037/jgo.2018.05.14

\section{Introduction}

Despite advances in cancer management, hepatocellular carcinoma (HCC) remains the sixth most common malignancy and the second leading cause of cancer-related death worldwide $(1,2)$. While surgical resection remains the mainstay of the curative treatment approach, patients with unresectable cancer or poor hepatic reserve secondary to chronic liver disease often cannot undergo this procedure. As such, multiple liver-directed treatment options are utilized including radioembolization of the lesion(s) (3). To assess the degree to which patients will tolerate these liver-directed treatments, various laboratory and scoring systems exist.

Child-Pugh (CP) is one of such scoring systems used to assess liver dysfunction in a variety of medical settings. Its progenitor, the Child score, emerged in the surgical setting in 1964 with the original criteria: serum bilirubin, serum albumin, presence of ascites, underlying neurological disorder, and nutritional status. The classification system split the patient population into three classes which 
Table 1 Criteria and scores for the Child-Pugh classification system

\begin{tabular}{|c|c|c|c|}
\hline Criteria & 1 point & 2 points & 3 points \\
\hline Serum albumin (g/dL) & $>3.5$ & $2.8-3.5$ & $<2.8$ \\
\hline Prothrombin time or INR & $<4.0 ;<1.7$ & $4.0-6.0 ; 1.7-2.3$ & $>6.0 ;>2.3$ \\
\hline Ascites & None & Mild (or controlled with medication) & Moderate to severe (or refractory to medication) \\
\hline
\end{tabular}

INR, international normalized ratio.

continues to be reflected in the modern day grouping of the $\mathrm{CP}$ classes. It was originally devised to assess hepatic reserve for patients with cirrhosis to identify suitable candidates for portal decompression. This publication represented the first recognized scoring system that systematically assessed hepatic function and used it for clinical decision-making (4).

After recognizing these limitations to the scoring system, and realizing that one strongly aberrant value may place a patient in the Child C class, Dr. Pugh revised the scoring system in 1973 . He elected to include prothrombin time, exclude nutritional status, modify the lab value cutoffs, and assign points for each factor rather than allow a single abnormal value to place the patient in a worse risk class. The outcome of this change represents the basis of the modern $\mathrm{CP}$ classification system. Despite these changes, the purpose of the scoring system remained unchanged; to help identify patients who would benefit from surgical intervention for esophageal hemorrhage in the setting of cirrhosis. While the original scoring system was used to guide surgical decision-making, it has been transposed to non-surgical fields of medicine to assess hepatic reserve in all settings of liver dysfunction (5). The CP classification system is comprised of several criteria that assess liver function (see Table 1). Broadening the spectrum of its use remains clinically relevant.

Multiple flaws have been identified with the design and use of the modern CP classification system. Although modified from previous criteria, the decision to pick those criteria was done with no significant statistical analyses. Along with this, the arbitrarily chosen cutoff values for these criteria have created an analytical bias. In this bias, a wide range of values may fall under the same CP score, despite representing significant differences in hepatic function. Two of these variables, the presence of hepatic encephalopathy and ascites, are graded subjectively with no concrete guideline that accounts for the severity and impact of pharmacologic management on the score of these criteria. Some of the criteria simply represent overlapping assessments of the liver function, such as ascites and serum albumin level. Finally, the CP system does not offer functional discrimination for patients with HCC outside of the setting of cirrhosis, as a majority of these patients fall into the CP A class (6). Given these issues, newer models for the assessment of liver function have been developed.

Johnson et al. developed the Albumin-Bilirubin (ALBI) model to serve as a purely objective marker of liver function (7). The formula was developed from a large Japanese dataset of patients where hepatitis $\mathrm{C}$ virus (HCV) was the predominant etiology of HCC. It was subsequently validated on HCC datasets from China (HBV), Spain, Boston, and United Kingdom (HCV and alcohol abuse), patients receiving sorafenib for unresectable HCC in two international trials, patients with cirrhosis but no HCC, and Japanese patients who had previously undergone HCC resection. They had determined that ALBI was able to achieve the same level of discrimination as the CP system. They were also able to validate the model further to show that within the CP A class, in fact, lie two distinct patient groups that have significant differences in survival outcomes which ALBI was able to discern. We aim to retrospectively assess Moffitt Cancer Center's outcome data for patients with $\mathrm{HCC}$ receiving radioembolization using both the $\mathrm{CP}$ and ALBI scoring systems.

\section{Methods}

\section{Patients}

With institutional board review approval, we retrospectively collected data from Moffitt Cancer Center patients with HCC who received yittrium-90 (Y-90) radioembolization between July 2009 and March 2014. Patients who were adults with unresectable HCC treated with Y-90 radioembolization were eligible in our analysis. As a 
standard of care, our patients had their liver function tests evaluated immediately prior to the radioembolization procedure, giving a consistent time point of information which was used to calculate the CP and ALBI values.

\section{Diagnosis}

HCC diagnosis was made by a multidisciplinary hepatology team using clinical and radiographic information with a confirmatory biopsy only performed when deemed medically necessary. HCC staging was performed based on the American Joint Committee on Cancer staging criteria (8). Patients deemed unresectable by our medical and surgical oncologists at multidisciplinary tumor board were evaluated for locoregional radiotherapy.

\section{Treatment and follow-up}

Radioembolization was performed using glass microspheres (TheraSphere; BTG International, West Conshohocken, PA, USA) by our staff interventional radiologists and radiation oncologists. Angiography and technetium-99m scintigraphy were used to estimate lung shunting, identify extrahepatic perfusion, and to perform coil embolization as deemed necessary. The treatment volume was measured on cross-sectional imaging. This was used to calculate the radiation activity based on the Y-90 formula $A(G b q)=$ [D $(G y) \times M(k g)] / 50$ where $A$ is the activity, $D$ the nominal target dose, and $M$ the liver mass for the planned target volume (PTV) (i.e., segment, lobe, or whole liver). All treatments were delivered in an outpatient setting (9-12). Interdisciplinary follow-up was set on a consistent 1-, 3-, 6-month basis.

Calculation of score values: The CP score was calculated as accepted from the modified system in 1973 (5). The subjective portions of the CP scores were ascertained from clinical notes on the day of or within the 4 weeks prior of the procedure. The ALBI score and grades were calculated as outlined by the ALBI development manuscript using the following formula (7) (Figure 1):

ALBI score $=\left(\log _{10}\right.$ bilirubin $\left.\times 0.66\right)+($ albumin $\times-0.085)$

Grade 1

Grade 2

$-1.39 \quad$ Grade 3

Figure 1 The ALBI grading spectrum. ALBI, Albumin-Bilirubin.
Where bilirubin is measured in $\mu \mathrm{mol} / \mathrm{L}$ and albumin is measured in $\mathrm{g} / \mathrm{L}$.

\section{Statistical analysis}

Non-parametric data were expressed using median value as the central descriptor (range). Overall survival rates were calculated by the Kaplan-Meier method and statistical significance was set at $\mathrm{P}<0.05$ using the Mantel-Cox Log Ranking analysis. All statistical calculations and survival curves were generated using SPSS version 23.2 (IBM, Armonk, New York 10504, USA).

\section{Results}

A total of 124 patients were identified to have received radioembolization for confirmed HCC. Median age at the time of treatment was 66 years (range, 45-86 years) with $99(79.8 \%)$ male patients and 25 (20.2\%) female patients. Median follow up was 10.1 months. A majority of the patients had no prior targeted therapy (102/124) and only a small group had prior targeted therapy (22/124). Treatment volumes consisted of involved lobes and segments depending on the clinical circumstances and no patient received whole liver Y-90 in one treatment. Typically, treatment was delivered, follow-up parameters were evaluated at 6-8 weeks and, if indicated, additional Y-90 was delivered several weeks later. Since the patients received segmental as well as lobar treatments, the median dose was higher at 131.0 Gy (range, 99.0-777.0 Gy). Patient characteristics are summarized in Table 2. Patients were initially stratified based on CP classes with class A $(\mathrm{n}=108)$ having a median survival of 13.10 months (range, 10.10-16.20 months; SD, 1.60), class B ( $\mathrm{n}=14)$ having a median survival of 8.40 months (range, 1.25-18.34 months; $\mathrm{SD}, 5.10)$, and class $\mathrm{C}(\mathrm{n}=2)$ having a median survival of 0.60 months (range, 0.62-2.86 months; SD, 1.58) (Figure 2). CP classes $\mathrm{A}$ and $\mathrm{B}$ had significantly better survival functions $\left(\mathrm{P}<0.0005, \chi^{2}=39.594, \mathrm{df}=2\right)$. Patients were then stratified into different ALBI grades with grade $1(n=50)$ having a median survival of 20.90 months (range, 13.50-28.30 months; SD, 3.80), grade $2(\mathrm{n}=71)$ having a median survival of 11.00 months (range, 8.40-13.70 months; SD, 1.40), and grade $3(n=3)$ having a median survival of 0.60 months (range, 0.62-2.86 months; SD, 1.58) (Figure 3). Lower 
Table 2 Characteristics of the patient cohort

\begin{tabular}{|c|c|}
\hline Characteristics & Values \\
\hline Age, median [range] (years) & $66[45-86]$ \\
\hline \multicolumn{2}{|l|}{ Gender, N } \\
\hline Male & 99 \\
\hline Female & 25 \\
\hline \multicolumn{2}{|l|}{ Current alcohol use, $\mathrm{N}$} \\
\hline Yes & 19 \\
\hline No & 105 \\
\hline \multicolumn{2}{|l|}{ Hepatitis B infection, $\mathrm{N}$} \\
\hline Yes & 8 \\
\hline No & 116 \\
\hline \multicolumn{2}{|l|}{ Hepatitis $\mathrm{C}$ infection, $\mathrm{N}$} \\
\hline Yes & 56 \\
\hline No & 68 \\
\hline \multicolumn{2}{|c|}{ Non-alcoholic steatohepatitis, $\mathrm{N}$} \\
\hline Yes & 7 \\
\hline No & 117 \\
\hline \multicolumn{2}{|l|}{ Newly diagnosed, $\mathrm{N}$} \\
\hline Yes & 28 \\
\hline No & 96 \\
\hline \multicolumn{2}{|l|}{ Extrahepatic disease, $\mathrm{N}$} \\
\hline Yes & 8 \\
\hline No & 116 \\
\hline \multicolumn{2}{|l|}{ Prior targeted therapy, $\mathrm{N}$} \\
\hline Yes & 22 \\
\hline No & 102 \\
\hline \multicolumn{2}{|l|}{ Prior surgery, $\mathrm{N}$} \\
\hline Yes & 12 \\
\hline No & 112 \\
\hline \multicolumn{2}{|c|}{ Prior radiofrequency ablation, $\mathrm{N}$} \\
\hline Yes & 11 \\
\hline No & 113 \\
\hline \multicolumn{2}{|c|}{ Prior external beam radiotherapy, $\mathrm{N}$} \\
\hline Yes & 1 \\
\hline No & 123 \\
\hline
\end{tabular}

Table 2 (continued)
Table 2 (continued)

\begin{tabular}{|c|c|}
\hline Characteristics & Values \\
\hline \multicolumn{2}{|c|}{ Prior chemoembolization, $\mathrm{N}$} \\
\hline Yes & 18 \\
\hline No & 106 \\
\hline \multicolumn{2}{|l|}{ Prior radioembolization, $\mathrm{N}$} \\
\hline Yes & 30 \\
\hline No & 94 \\
\hline \multicolumn{2}{|l|}{ Treatment volume, $\mathrm{N}$} \\
\hline Lobar & 87 \\
\hline Segmental & 37 \\
\hline $\begin{array}{l}\text { Median dose received, } \\
\text { median (range) (Gy) }\end{array}$ & $131.0(99.0-777.0)$ \\
\hline $\begin{array}{l}\text { Medium volume treated, } \\
\text { median (range) }(\mathrm{mL})\end{array}$ & $820.0(50.0-2,630.0)$ \\
\hline \multicolumn{2}{|l|}{ Child-Pugh class } \\
\hline$A$ & 108 \\
\hline $\mathrm{B}$ & 14 \\
\hline $\mathrm{C}$ & 2 \\
\hline \multicolumn{2}{|l|}{ ALBI grade } \\
\hline 1 & 50 \\
\hline 2 & 71 \\
\hline 3 & 3 \\
\hline
\end{tabular}

ALBI, Albumin-Bilirubin.

ALBI grades were significantly associated with better overall survival $\left(\mathrm{P}<0.0005, \chi^{2}=49.371, \mathrm{df}=2\right)$. All CP A patients were re-grouped based on their ALBI grades to find that ALBI grade $1(n=48)$ had a median survival of 20.90 months (range, 13.60-28.10 months; SD, 3.70) and grade $2(\mathrm{n}=60)$ had a median survival of 11.00 months (range, 8.5013.50 months; SD, 1.30) (Figure 4). Lower ALBI grades within the $\mathrm{CP}$ A population were associated with significantly better survival outcomes $\left(\mathrm{P}=0.002, \chi^{2}=9.385, \mathrm{df}=1\right)$.

\section{Discussion}

When using the traditional CP classification system, a majority of patients are categorized as the lowest risk category: CP class A. Only a small portion of patients were CP B and only two were CP C. With this representation 


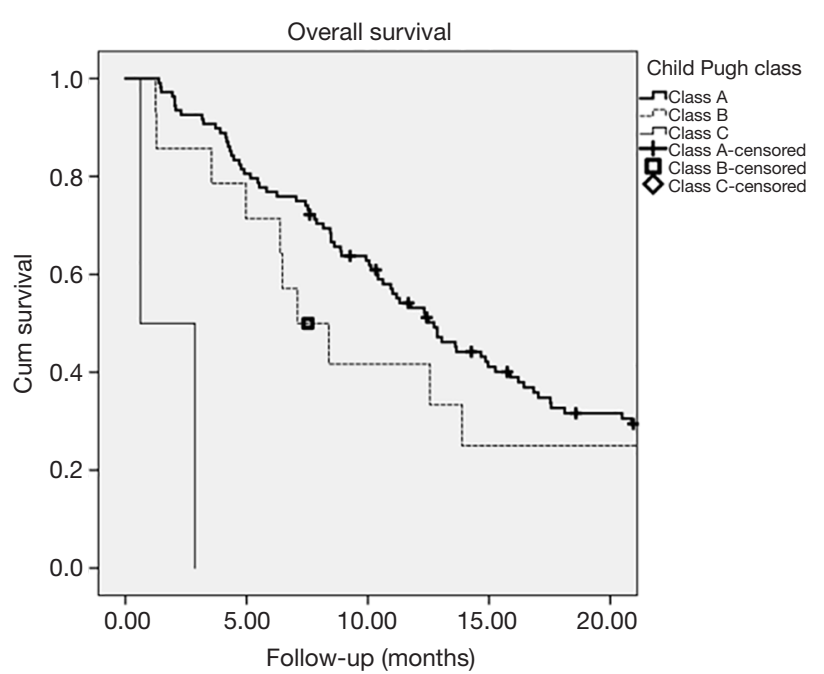

Figure 2 Kaplan-Meier survival plot of the entire patient cohort stratified by the Child-Pugh classification system.

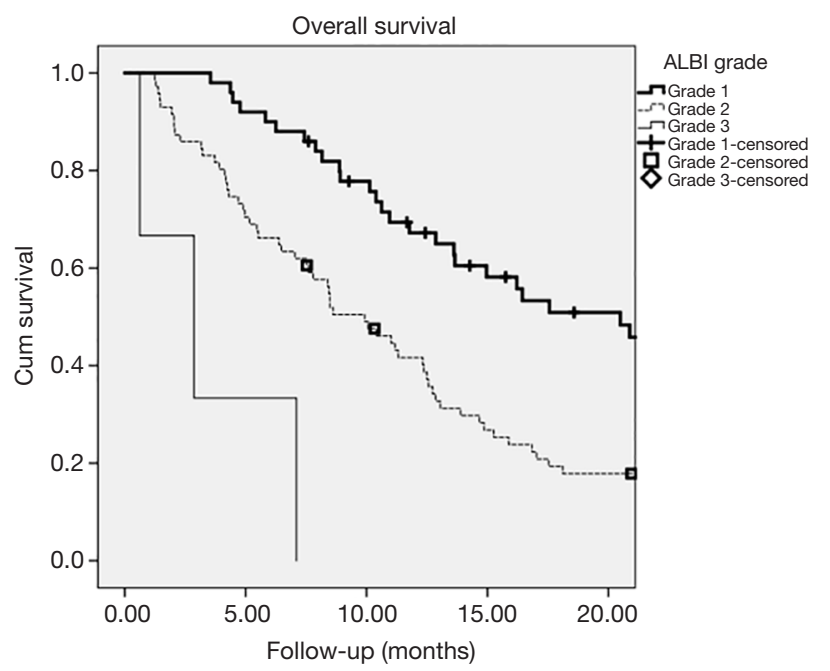

Figure 3 Kaplan-Meier survival plot of the entire patient cohort stratified by the Albumin-Bilirubin (ALBI) grading system.

of the data, the median survival difference between the CP A and CP B classes was 4.7 months. Significantly more patients were graded as ALBI 2 compared to CP B and the median survival difference between ALBI 1 and ALBI 2 was 9.9 months. Once again, only two patients were graded as ALBI 3. This comparison between the median survival differences of the first two groups within each grading system highlights the importance of moving away from the CP system. As a majority of patients are graded as CP A, less information can be drawn from their

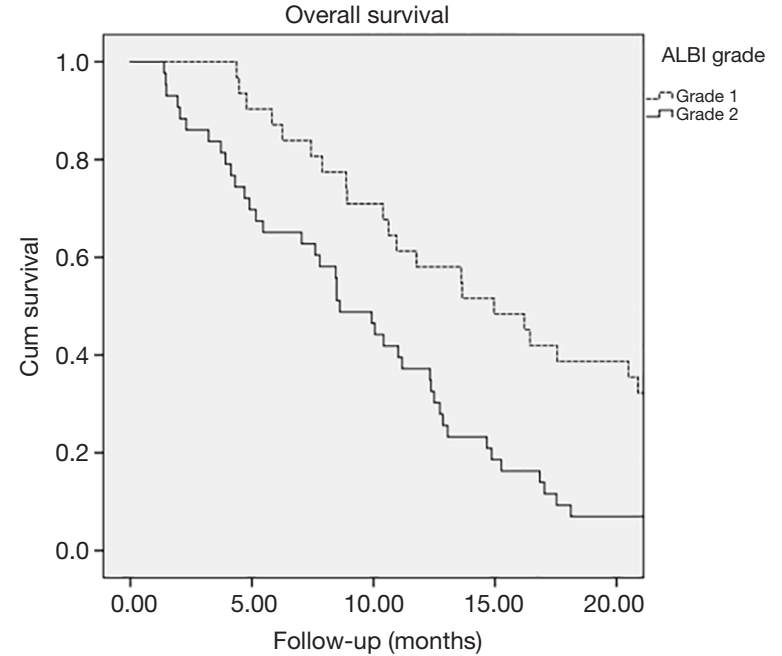

Figure 4 Kaplan-Meier survival plot of the Child-Pugh class A subcohort stratified by the Albumin-Bilirubin (ALBI) grading system.

clinical performance and anticipated outcomes. This lost stratification of patients is observed within the ALBI system which demonstrated a larger survival difference between the first two grading groups. Reclassifying the CP A patients in the ALBI system, the median survival difference between ALBI 1 and ALBI 2 was found to be 9.9 months. Not surprisingly, this number correlates to the initial ALBI 1 and 2 survival differences, as the CP A class represented a majority of the patients in this study. Little information can be drawn from the CP C and ALBI 3 groups as these had minimal patients and statistically insignificant outcomes. This is a reassuring finding as it demonstrates our clinically appropriate selection of patients for radioembolization.

Our study is not the first to demonstrate the utility of the ALBI grade. Prior publications have proposed the use of the ALBI system as a more accurate prognostic model and assessment of patients' liver dysfunction. It has been found to be valuable as a predictor of outcomes after liver resection for HCC, a prognostic scoring system for primary biliary cirrhosis, for prognosticating outcomes in patients failing sorafenib, discriminating survival after transarterial chemoembolization (TACE) or radioembolization for HCC, and improving existing HCC staging systems (13-18).

Several groups have modified preexisting staging systems with the incorporation of the ALBI grade to improve the prognostication abilities of said staging systems. Chan et al. evaluated the prognostic performance of the Cancer of the Liver Italian Program (CLIP) in a large cohort of 1973 Chinese patients with HCC. They compared the CP-CLIP, 
ALBI-CLIP, and nine other grading systems using the homogeneity likelihood Chi-square, C-index, and corrected Akaike information criterion. Based on these three criteria, they found both the CP-CLIP and ALBI-CLIP to be the most powerful staging systems out of the 11 that exist. ALBI-CLIP provided comparable prognostic performance compared to CP-CLIP with comparable scores within the ALBI-CLIP having improved median survival (16).

Our study focused on evaluating the CP classes as a whole and did not break each class into their numeric subgroups. The lack of sub-class refinement may have obscured CP's ability to better discriminate outcomes in the patient sub-populations. However, Hiraoka et al. looked into the $\mathrm{CP}$ classes more closely and divided the patients of CP A scores 5 and 6 into ALBI grades separately. Of their 1,285 CP A5 patients, 858 (66.8\%) were ALBI 1 and 427 (33.2\%) were grade 2. Of their 586 CP A6 patients, 53 (9.0\%) were ALBI 1 and 533 (91.0\%) were ALBI 2. They also found the percentage of ALBI 2 patients amongst the CP A6 class (91.0\%) was similar to that amongst CP B7 class $(91.8 \%)$. These findings demonstrate the inability of $\mathrm{CP}$ to distinguish the difference between the border of $\mathrm{CP}$ classes $\mathrm{A}$ and $\mathrm{B}$, which was confirmed in our study by the migration of a significant portion of our CP A patients to ALBI 2 grade. Their study further supports the use of ALBI for a higher degree of discrimination as a significant number of CP A5 patients migrated to ALBI grade 2 (19).

For our radioembolization study, only $17.8 \%$ of our patients were previously treated with sorafenib. This questions the reliability of ALBI in patients with prior sorafenib use as hepatotoxicity is a known adverse effect of the medication $(20,21)$. The resulting hypoalbuminemia could drastically alter the ALBI grade and place patients in worse risk categories. Other studies have further evaluated the use of ALBI in patients that have received sorafenib. Edeline et al. evaluated 905 patients with HCC treated with sorafenib from 17 centers in the United Kingdom and France. Their ALBI grade 2 patients were found to be comprised of CP A5 (33.3\%), CP A6 (44.3\%), and CP >A6 (22.4\%). They found the median overall survival (OS) in CP classes A5, A6, and > A6 was 10.2, 7.0, and 3.6 months, respectively $(\mathrm{P}<0.001)$. Similarly, the median OS in ALBI grades 1,2 , and 3 was $10.9,6.6$, and 3.0 months, respectively $(\mathrm{P}<0.001)$. Although they found the discriminatory abilities of CP and ALBI to be similar in the CP A class, CP had better performance in the overall population. Their study once again demonstrates that the CP A class contains a heterogeneous population which can be further stratified by the ALBI grade (1).

In further support of using ALBI in patients treated with sorafenib, Lee et al. evaluated the creation of an ALBI-based criterion for enrolling sorafenib-failed HCC patients in second-line clinical trials. They used patients who developed progressive disease (PD) from their 404 consecutive HCC patients receiving sorafenib. These PD patients were randomized in a 1:1 fashion to the derivation and validation cohorts. The first group was used to create the ALBI-progressive-disease (ALBI-PD) criteria and the second was used to validate it. They found that CP A patients in the derivation and validation groups with $\mathrm{PD}$ had post-progression survival (PPS) of 9.6 and 9.7 months, respectively $(\mathrm{P}<0.001)$. PPS for patients falling within their ALBI-PD criteria was 3.6 months and 4.9 months if the subjects were beyond their ALBI-PD criteria $(\mathrm{P}=0.005)$. Additionally, the ALBI-PD criteria discriminated the PPS among patients in the CP A5-B7 classes with significance. They concluded that their ALBI-PD criteria can differentiate PPS and stratify patients with advanced HCC for second-line clinical trials (14).

\section{Conclusions}

In conclusion, our retrospective study supports the use of the ALBI grading system to assess liver function with more discrimination compared to the CP classes in the setting of radioembolization for patients with HCC. This suggests that the ALBI grade may be a useful prognostic tool for liver directed therapy patient selection in this setting. No conclusions can be drawn regarding ALBI grade 3 and $\mathrm{CP}$ class $\mathrm{C}$ patients as there were too few in our study to analyze.

\section{Acknowledgements}

None.

\section{Footnote}

Conflicts of Interest: The authors have no conflicts of interest to declare.

Ethical Statement: The study was approved by University of South Florida Moffitt Institutional Review Board (IRB No. Pro00003382) and Informed consent was not obtained 
for this retrospective study as the IRB approved a waiver of signed authorization.

\section{References}

1. Edeline J, Blanc JF, Johnson P, et al. A multicentre comparison between Child Pugh and Albumin-Bilirubin scores in patients treated with sorafenib for Hepatocellular Carcinoma. Liver Int 2016;36:1821-8.

2. Torre LA, Bray F, Siegel RL, et al. Global cancer statistics, 2012. CA Cancer J Clin 2015;65:87-108.

3. Benson AB 3rd, D'Angelica MI, Abbott DE, et al. NCCN Guidelines Insights: Hepatobiliary Cancers, Version 1.2017. J Natl Compr Canc Netw 2017;15:563-73.

4. Child CG, Turcotte JG. Surgery and portal hypertension. Major Probl Clin Surg 1964;1:1-85.

5. Pugh RN, Murray-Lyon IM, Dawson JL, et al. Transection of the oesophagus for bleeding oesophageal varices. Br J Surg 1973;60:646-9.

6. Johnson PJ, Berhane S, Satomura S, et al. An international collaborative study assessing the role of aetiology and stage in survival in HCC: Implications for screening. Presented at International Liver Congress London, European Association for the Study of the Liver; April 9-13; London, United Kingdom, 2014.

7. Johnson PJ, Berhane S, Kagebayashi C, et al. Assessment of liver function in patients with hepatocellular carcinoma: a new evidence-based approach-the ALBI grade. J Clin Oncol 2015;33:550-8.

8. Edge SB, Compton CC. The American Joint Committee on Cancer: the 7th edition of the AJCC cancer staging manual and the future of TNM. Ann Surg Oncol 2010;17:1471-4.

9. Murthy R, Nunez R, Szklaruk J, et al. Yttrium-90 microsphere therapy for hepatic malignancy: devices, indications, technical considerations, and potential complications. Radiographics 2005;25 Suppl 1:S41-55.

10. Salem R, Lewandowski RJ, Atassi B, et al. Treatment of unresectable hepatocellular carcinoma with use of $90 \mathrm{Y}$ microspheres (TheraSphere): safety, tumor response, and survival. J Vasc Interv Radiol 2005;16:1627-39.

11. Salem R, Thurston KG. Radioembolization with yttrium-90 microspheres: a state-of-the-art brachytherapy treatment for primary and secondary liver malignancies: part 3: comprehensive literature review and future direction. J Vasc Interv Radiol 2006;17:1571-93.

12. Abuodeh Y, Naghavi AO, Ahmed KA, et al. Prognostic value of pre-treatment F-18-FDG PET-
CT in patients with hepatocellular carcinoma undergoing radioembolization. World J Gastroenterol 2016;22:10406-14.

13. Wang YY, Zhong JH, Su ZY, et al. Albumin-bilirubin versus Child-Pugh score as a predictor of outcome after liver resection for hepatocellular carcinoma. Br J Surg 2016;103:725-34.

14. Lee PC, Chen YT, Chao Y, et al. Validation of the albumin-bilirubin grade-based integrated model as a predictor for sorafenib-failed hepatocellular carcinoma. Liver Int 2018;38:321-30.

15. Hickey R, Mouli S, Kulik L, et al. Independent Analysis of Albumin-Bilirubin Grade in a 765-Patient Cohort Treated with Transarterial Locoregional Therapy for Hepatocellular Carcinoma. J Vasc Interv Radiol 2016;27:795-802.

16. Chan AW, Chong CC, Mo FK, et al. Incorporating albumin-bilirubin grade into the cancer of the liver Italian program system for hepatocellular carcinoma. J Gastroenterol Hepatol 2017;32:221-8.

17. Chan AW, Kumada T, Toyoda $\mathrm{H}$, et al. Integration of albumin-bilirubin (ALBI) score into Barcelona Clinic Liver Cancer (BCLC) system for hepatocellular carcinoma. J Gastroenterol Hepatol 2016;31:1300-6.

18. Chan AW, Chan RC, Wong GL, et al. New simple prognostic score for primary biliary cirrhosis: Albuminbilirubin score. J Gastroenterol Hepatol 2015;30:1391-6.

19. Hiraoka A, Kumada T, Michitaka K, et al. Usefulness of albumin-bilirubin grade for evaluation of prognosis of 2584 Japanese patients with hepatocellular carcinoma. J Gastroenterol Hepatol 2016;31:1031-6.

20. Liu L, Chen H, Wang M, et al. Combination therapy of sorafenib and TACE for unresectable HCC: a systematic review and meta-analysis. PLoS One 2014;9:e91124.

21. Gebski V, Gibbs E, Gandhi M, et al. VESPRO: An Individual Patient Data Prospective Meta-Analysis of Selective Internal Radiation Therapy Versus Sorafenib for Advanced, Locally Advanced, or Recurrent Hepatocellular Carcinoma of the SARAH and SIRveNIB Trials. JMIR Res Protoc 2017;6:e17.

Cite this article as: Mohammadi $\mathrm{H}$, Abuodeh $\mathrm{Y}$, Jin W, Frakes J, Friedman M, Biebel B, Choi J, El-Haddad G, Kis B, Sweeney J, Hoffe S. Using the Albumin-Bilirubin (ALBI) grade as a prognostic marker for radioembolization of hepatocellular carcinoma. J Gastrointest Oncol 2018;9(5):840-846. doi: 10.21037/jgo.2018.05.14 